\title{
Consolidating evidence on the effectiveness of interventions promoting fruit and vegetable consumption: an umbrella review
}

Luke Wolfenden ${ }^{1,2^{*}}$, Courtney Barnes ${ }^{1}$, Cassandra Lane ${ }^{1}$, Sam McCrabb ${ }^{1}$, Hannah M. Brown ${ }^{1}$, Sarah Gerritsen ${ }^{2,3}$, Simon Barquera ${ }^{2,4}$, Lesly Samara Véjar ${ }^{4}$, Ana Munguía ${ }^{4}$ and Sze Lin Yoong ${ }^{5}$

\begin{abstract}
Background: The overarching objective was to examine the effectiveness of intervention strategies to promote fruit and vegetable consumption. To do this, systematic review evidence regarding the effects of intervention strategies was synthesized; organized, where appropriate, by the setting in which the strategies were implemented. Additionally, we sought to describe gaps in the review of evidence; that is, where evidence regarding the effectiveness of recommended policy actions had not been systematically synthesised.

Methods: We undertook a systematic search of electronic databases and the grey literature to identify systematic reviews describing the effects of any intervention strategy targeting fruit and/or vegetable intake in children or adults of any age.

Results: The effects of 32 intervention strategies were synthesised from the 19 included reviews. The strategies were mapped across all three broad domains of the NOURISHING framework (i.e. food environment, food system and behaviour change communication), but covered just 14 of the framework's 65 sub-policy areas. There was evidence supporting the effectiveness of 19 of the 32 intervention strategies. The findings of the umbrella review suggest that intervention strategies implemented within schools, childcare services, homes, workplaces and primary care can be effective, as can eHealth strategies, mass media campaigns, household food production strategies and fiscal interventions.

Conclusions: A range of effective strategy options are available for policy makers and practitioners interested in improving fruit and/or vegetable intake. However, the effects of many strategies - particularly those targeting agricultural production practices, the supply chain and the broader food system - have not been reported in systematic reviews. Primary studies assessing the effects of these strategies, and the inclusion of such studies in systematic reviews, are needed to better inform national and international efforts to improve public health nutrition.
\end{abstract}

Trial registration: The review protocol was deposited in a publicly available Open Science framework prior to execution of the search strategy. https://osf.io/unj7x/.

Keywords: Nutrition policy, Recommended dietary allowances, Diet, Public health, Health promotion

\footnotetext{
* Correspondence: Luke.Wolfenden@health.nsw.gov.au

${ }^{1}$ School of Medicine and Public Health, The University of Newcastle, Hunter New England Population Health, Locked Bag 10 Wallsend NSW, Newcastle, NSW 2287, Australia

${ }^{2}$ Corporación Actuemos, Santiago, Chile

Full list of author information is available at the end of the article
}

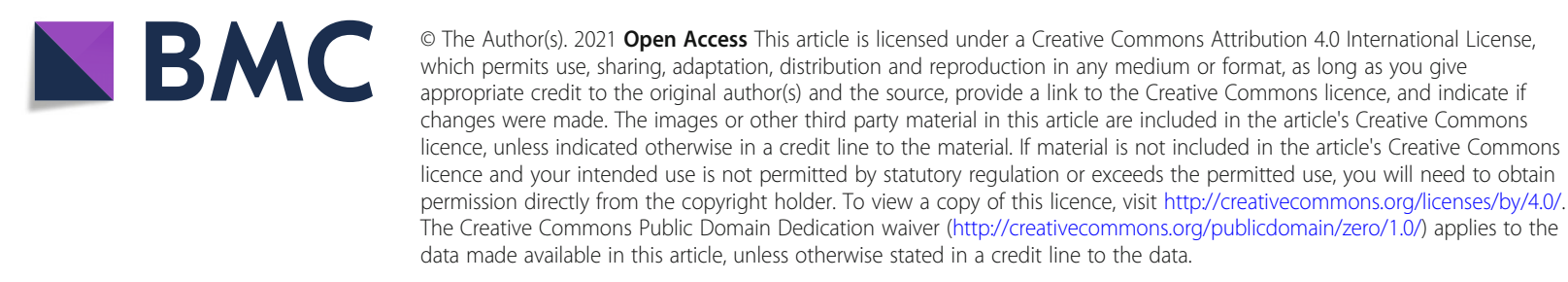




\section{Background}

Low fruit and vegetable consumption are a modifiable risk factor that is contributing to the rising international burden of non-communicable diseases [1, 2]. In 2017, 3.9 million deaths worldwide were attributed to inadequate fruit and vegetable intake [3]. Adequate intake of fruits and vegetables reduces the risk of a variety of chronic health conditions including hypertension, coronary heart disease, stroke [4] and type 2 diabetes [5]. The health promoting properties of fruits and vegetables can be attributed to their concentrations of bioactive compounds, including vitamins, minerals, antioxidants and fibre [6]. The concentration of these compounds may differ between fruits and vegetables, with fruits typically containing more dietary sugars, and vegetables more protein and fibre [6]. Although certain types of fruits or vegetables may be particularly beneficial for particular health outcomes - for example, cruciferous vegetables may reduce the risk of a number of specific cancers [7-9] - intake of both fruits and vegetables is recommended to promote good health [10].

The World Health Organization (WHO) recommends a combined consumption of at least $400 \mathrm{~g}$ (g) of fruits and vegetables per day [3]. However, current global fruit and vegetable intakes fall short of the $\mathrm{WHO}$ recommendations. A systematic analysis of 266 country-specific nutrition surveys worldwide found that, in 2010, global fruit intake was $81.3 \mathrm{~g} /$ day, with only two countries having mean intakes of at least $300 \mathrm{~g} /$ day [11]. There is a similar situation with youth - Global School-Based Student Health Survey data from 2004 to 2013 found that less than $30 \%$ of adolescents from 49 low- and middle-income countries (LMIC) met WHO minimum recommended levels of intake for fruits and vegetables [12].

Improving population intakes of fruits and vegetables represents a considerable challenge. The determinants of intake are complex, and nest within a dynamic food system where there is interaction of factors such as food production, supply and affordability, access, food environments and individual behaviours [13]. Several of these determinants, specifically those that relate to supply, access and affordability, are particularly acute in LMIC [13]. A range of global plans have been developed to prompt action to increase fruit and vegetable intake, including the WHO Global Strategy on Diet, Physical Activity and Health and, more recently, the WHO Global Action Plan for the Prevention and Control of Non Communicable Diseases (NCDs) 2013-2020, and the United Nations (UN) Decade of Action on Nutrition 2016-2025. Similarly, the NOURISHING framework of the World Cancer Research Fund International (WCRF) has been established to guide national efforts to improve public health nutrition. The framework specifies a range of interventions across 10 key policy areas in three key domains: food environment (i.e. food labelling standards), food system (i.e. supply chain actions) and behavioural change communication (i.e. nutrition counselling) $[14,15]$. The framework was developed following a meeting of international experts; it draws on previously developed frameworks and aligns with international policy options to improve public health nutrition. It is also intended to facilitate the reporting and monitoring of policy actions internationally.

To date, government action to improve fruit and vegetable consumption has been variable. The Global Nutrition Policy Review found that just 63\% of 167 countries with national nutrition polices included goals, targets or indicators focused on improving fruit and vegetable intake; also, initiatives to promote fruit and vegetable consumption varied considerably by WHO region [16]. The report found that the proportion of countries with schoolbased fruit and vegetable schemes had decreased markedly over the past decade, but among the WHO regions it was highest in Europe (51\%), and lowest in Africa (13\%) and the Eastern Mediterranean (16\%). A 2018 audit of the WCRF NOURISHING database, which catalogues government nutrition policies and actions globally, reported 168 polices specifically designed to improve fruit and vegetable intake [13]. However, these policies focused almost entirely on two of the 10 domains of the framework: offering healthy food and setting standards in institutions and settings, and informing people about food and nutrition through public awareness initiatives [13]. Such findings are similar to earlier systematic reviews of nutrition policies in LMIC, which have reported that most global nutrition activities to improve fruit and vegetable intake are focused on public education and demonstrations [17].

To maximise the potential impact of investment in initiatives to improve fruit and vegetable intake, it is recommended that evidence be used to influence decisionmaking in health policy and practice. Rigorous systematic reviews aim to identify, capture and consider all relevant evidence $[18,19]$. As such, the use of such reviews is recommended as the basis of health policy and practice decisions [20]. There is an ever-increasing pool of primary studies investigating the effects of fruit and vegetable intervention strategies; in recent years, numerous systematic reviews have been undertaken to synthesise this evidence [21-26]. Managing the information presented in large numbers of systematic reviews can be challenging for decision-makers. Unlike conventional systematic reviews, which synthesise the findings of individual primary studies, overviews of systematic reviews (also known as 'umbrella' reviews) can help to overcome these challenges by assessing and consolidating the findings of systematic reviews and meta-analyses [27]. Umbrella reviews provide a means of rapidly and efficiently synthesising evidence from a broad body of research, $[27,28]$ and can assist decision-makers in choosing from different intervention strategies; thus, they are frequently 
used as the basis for health policy and guideline development [29-32]. Umbrella reviews are also useful to identify gaps where policy or practice is being recommended in the absence of evidence (or contrary to evidence), or where beneficial intervention strategies exist but are not being routinely delivered.

Given the importance of fruit and vegetable intake to human health, and the breadth of strategies suggested to improve intake, we conducted an umbrella review to inform a background paper for the 2020 Food and Agriculture Organization of the UN (FAO)/WHO International Workshop on Vegetables and Fruits for Food and Quality of Life. The workshop seeks to support public sector organisations to develop national policies, strategies, regulatory frameworks and other intervention strategies, to improve population fruit and vegetable intake. The overarching objective is to examine the effectiveness of intervention strategies to promote fruit and vegetable consumption. To do this, we synthesized systematic review evidence regarding the effects of intervention strategies, organized (where appropriate) by the setting in which they were implemented. We also sought to describe gaps in the review of evidence (i.e. areas where evidence regarding the effectiveness of recommended policy actions had not been systematically synthesised). In both synthesis of evidence and identification of gaps, we used the recommended policy areas identified in the WCRF NOURISHING framework.

\section{Methods}

The review was guided by recommendations for the conduct of umbrella reviews from the Joanna Briggs Institute [33] and the Cochrane Handbook [34]. The review protocol was deposited in a publicly available Open Science framework prior to execution of the search strategy (https:// osf.io/unj7x/) [35]. The findings of the review are reported based on suggestions in the protocol for Preferred Reporting Items for Overviews of Reviews (PRIOR) [36].

\section{Inclusion and exclusion criteria}

We included systematic reviews that assessed the effectiveness of intervention strategies on a measure of fruit and vegetable intake. We included one systematic review per intervention strategy identified. Consistent with recommendations in the Cochrane Handbook, where multiple reviews report the effects of the same intervention strategy, we selected the most recent high-quality review for inclusion. This approach meant that the number of systematic reviews included could not exceed the number of strategies reported in this umbrella review. However, in some instances, a single review was included that reported on the effects of multiple intervention strategies.

\section{Design}

We included systematic reviews with or without metaanalysis that described the effectiveness of any intervention strategies on a measure of fruit and/or vegetable intake in children or adults. Initially, we included only reviews of controlled trials; however, following initial citation screening, we modified the criteria to include reviews of any prospective evaluation design (with or without a parallel control or comparison group). The aim of this modification was to provide greater opportunity for the inclusion of reviews reporting the effects of macrolevel intervention strategies (e.g. natural experiments following fiscal policy change) that may not be amenable to group allocation. All citations were rescreened using this modified criterion to ensure consistency with the screening method.

We excluded reviews comprising solely qualitative research, because they do not provide quantitative estimates of the effects of intervention strategies. Reviews published before 2011 were also excluded (as per Joanna Briggs Institute guidance), because those published in the past 10 years represent the contemporary evidence base and will capture primary research conducted over the previous 30 or so years [27].

Also excluded were reviews of intervention strategies on population groups selected on the basis of preexisting comorbidities (e.g. those with type 2 diabetes, hypertension or cancer); reviews focused on strategies that targeted the treatment or management of eating disorders (e.g. anorexia nervosa or bulimia) or other diseases; reviews of intervention strategies undertaken in laboratories or other simulated contexts (e.g. laboratorybased experiments of infant feeding practices); reviews in which intervention strategies were defined only by the population group targeted and not characterised by setting, delivery modality or content; and reviews examining intervention mechanisms (e.g. theory-based mechanisms of effect), or in which the effects of intervention components were synthesised at the level of individual behaviour change techniques.

\section{Outcomes}

The primary outcome for the umbrella review was fruit and/or vegetable intake (referred to here as 'fruit and vegetable intake' unless otherwise specified), assessed via self-report, observational, biochemical or other measures, or via objective measures of fruit and vegetable purchases (as an accepted proxy). Specifically, fruit and vegetable intake is measured in, for example, grams, portions or serves, assessed using measures such as food diaries, dietary recalls, food frequency questionnaires, observation or other approaches (e.g. measures of plate waste or photographs). Before undertaking the search, we also included objective measures of purchases of 
fruits and vegetables (e.g. those supplied by food outlets or supermarkets) as a proxy for dietary intake. Such outcomes are more likely to be used in evaluations of intervention strategies at policy or food system level, and represent a reliable surrogate measure of intake. To be included, systematic reviews must have synthesised the effects of strategy types on any measure of fruit and vegetable intake across the included reviews, in narrative or quantitative form (e.g. meta-analysis). We excluded reviews that included primary studies that reported on fruit and vegetable outcomes as part of other dietary measures (e.g. total diet quality), but did not explicitly synthesis across primary studies. We also excluded measures of behavioural intention, preference or liking for fruits or vegetables.

\section{Search strategy}

A search of peer reviewed and grey literature was undertaken on 11 June 2020 in the following bibliographic databases: MEDLINE, Embase, CINAHL, The Cochrane Library, Scopus and Academic Search Ultimate. We combined terms for 'intervention strategy', 'systematic reviews' and 'fruits and vegetables', and limited the search to the dates 2011 to 11 June 2020. Search strategies developed in MEDLINE were adapted for other databases by an information specialist (see Additional File for the detailed search strategy). We also searched all publications listed in the WHO e-Library of Evidence for Nutrition Actions (eLENA), and the WCRF NOURISHING website for any additional systematic reviews on fruit and vegetable intake; conducted targeted Google Scholar searches; and searched for grey literature using the search engine 'Google'. Finally, we sent compiled lists of reviews identified through database searches to experts in the field of nutrition and public health from FAO and WHO, to identify any additional potentially eligible reviews.

\section{Review selection and data extraction}

A single reviewer (LW) initially screened all citations, and excluded all clearly ineligible citations based on title. The remaining titles and abstracts were screened in duplicate $(\mathrm{CB}, \mathrm{CL})$ for eligibility. The full texts of all potentially relevant reviews were obtained and assessed against the inclusion criteria in duplicate (CB, CL). Manuscripts published in non-English languages were translated using 'Google Translate' and, if manuscripts were eligible for inclusion, a native language reviewer was sought to assist with screening and extraction. Disagreement regarding the eligibility of a review was resolved by discussion and consensus, or by consultation with an additional reviewer (LW).

Data were extracted independently and in duplicate by review authors (CB, CL, LW). The data extracted included the following information recommended by the Joanna Briggs Institute for the conduct of umbrella reviews: [27] citation details, objectives of the included review, review eligibility criteria (e.g. population, intervention, comparison and outcome characteristics, as well as setting or context), number of databases (including grey literature) sourced and searched, date range of database searching, number of included primary studies, instrument used to appraise the quality of primary studies and the rating of their quality, fruit and vegetable outcomes and effects reported by intervention strategy type and comparison, and method of synthesis or analysis. Consistent with recommendations for umbrella reviews, [27] extraction and presentation of findings and results was limited to those presented by the included systematic reviews (i.e. primary studies were not re-analysed).

Where a review reported separate syntheses of the effects of different intervention strategies, we extracted information describing the effects of each synthesis. If two or more reviews reported the effects of the same intervention strategies, we included only the findings of the most recent high-quality review. To do this, we assessed all reviews with a search date within two years of the most recent review, and selected the review with the highest quality rating. This meant that, for each intervention strategy, we included and reported only the findings of syntheses in the most contemporary and rigorous reviews available, and thus reduced the risk of any potential bias from overlapping reviews and primary studies.

Where reviews examined the effects of the same intervention strategy but in mutually exclusive population groups - for example, children and adults - we reported the effects in both groups. However, where reviews were undertaken on a population group and a subset of a population group - for example, children and girls only - we included only the more inclusive sample (i.e. in this example, children rather than girls only).

\section{Assessment of methodological quality of included reviews}

We assessed the methodological quality of included reviews independently and in duplicate (SMc, $\mathrm{HB}$ ), using the critical appraisal tool developed by the Joanna Briggs Institute [27, 37]. Discrepancies between reviewer assessments were resolved via consensus. The checklist requires assessment of the review by authors against 11 methodological criteria, each scored as 'yes', 'no', 'unsure' or 'not applicable'. Additional file 2 provides details on how the scoring system was applied and details of the specific checklist items. Consistent with previous umbrella reviews, [37] we defined reviews as 'low quality' where 33\% or less of the criteria were met, 'medium quality' where $34-66 \%$ of the criteria were met, and 'high quality' where $67 \%$ or more of the criteria were met. Criteria deemed 'not applicable' were not included in the denominator in assessments of review quality [37]. 


\section{Data analysis}

We used basic frequencies to describe the search results; described excluded and included reviews in line with the Preferred Reporting Items for Systematic Reviews and Meta-Analyses (PRISMA) guidance [38]; and narratively described the characteristics of included reviews. Quality assessments of reviews are presented in tabular form for each included review. We included reviews employing both quantitative and narrative syntheses.

To describe the effectiveness of the intervention strategies we reported, where available, the point estimates of effect, measures of variability, $p$-values and measures of heterogeneity in any included meta-analysis. Where the included reviews had narratively synthesised intervention strategies, we extracted and reported statements within the review that best summarised their overall effects. Summary statements were agreed on by two authors $(\mathrm{CB}, \mathrm{CL}$ or LW). Where reviews used both a formal measure of risk of bias or assessment of the quality of primary studies (e.g. Cochrane Risk of Bias tool), [39] and an evidence grading system, such as Grading of Recommendations, Assessment, Development and Evaluation (GRADE), [40] we reported findings from the grading system. This was because the evidence grading systems consider both primary study quality or risk of bias as well as other attributes to describe the overall body of evidence, and are recommended for use in health decision-making [41].

We used the WCRF NOURISHING framework [42] to describe evidence gaps, by comparing policy areas recommended for action in the framework to the effects of interventions strategies synthesised in the included reviews. Specifically, two authors (CB, CL) mapped descriptions of the intervention strategies synthesised in the included systematic reviews to the framework, based on descriptors provided on the WCRF website [42]. The WCRF NOURISHING framework covers a comprehensive package of policies to promote healthy eating across three broad domains: food environment, food system and behaviour change communication. Governments are encouraged to implement initiatives across each of the 10 evidence-informed policy areas (and associated subpolicy areas). Although the framework has a focus on actions to improve healthy eating overall, it has previously been used to characterise fruit and vegetable initiatives globally, [13] and many of the proposed policy areas could conceivably affect intake. Where reviews synthesized the effects of intervention strategies that exclusively aligned with a discrete NOURISHING sub-policy area, we denoted them as being 'directly mapped' to that policy area in the framework. Where reviews assessed the effects of intervention strategies that included related components inclusive of but not restricted to a policy area, we denoted them as being 'indirectly mapped' to that policy area. We also listed reviews of intervention strategies that could not be mapped to any sub-policy area of the framework. Finally, we described, broadly, the effects of intervention strategies mapped to each area of the framework.

\section{Results}

A total of 3588 records were identified through database searching, with an additional 115 identified through targeted Google Scholar searches. After deduplication, and full text assessment, 46 reviews reporting the effects of 32 intervention strategies were identified. Following an assessment of review search date and quality (selecting the higher quality review where more than one review reported the effects of the same intervention strategy), 19 systematic reviews reporting the effects of 32 intervention strategies were ultimately included (Fig. 1).

\section{Characteristics of included reviews}

Characteristics of included reviews are described in Table 1. The reviews were published between 2011 and 2020. The number of primary studies within the included reviews ranged from 13 to 120 . Two reviews included only primary studies conducted in the United States (US) [52, 53] and one review was restricted to LMIC [49]. Of the 19 reviews, four included only randomised controlled trials (RCTs) or cluster RCTs, [23, 46, $54,58]$ and seven undertook pooled quantitative analyses [23, 26, 44, 46, 47, 54, 55]. Eight reviews included primary studies undertaken in children and adolescents only (between 1 and 18 years), [22, 23, 46, 51, 54, 55, 57, $58]$ two were in adults ( $>18$ years) only, $[50,56]$ nine included all ages, [26, 43-45, 47, 49, 52, 53] and one review did not specify ages included but gave the population as workers [48].

\section{Quality assessment of included reviews}

Additional file 2 describes the outcome of the quality assessment of included reviews. Most of the reviews were of a high quality $(n=12),[23,26,44-46,48,50,53-57]$. Seven reviews met $34-66 \%$ of the critical appraisal criteria (CA) (i.e. scored 'yes') and received an overall medium quality assessment $[22,43,47,49,51,52,58]$. The primary criteria for which included reviews were downgraded were inadequate resources to search for potentially eligible primary studies (i.e. the reviews used inappropriate databases, searched two or fewer databases, or did not search the grey literature search) (CA4) (9 reviews).

\section{Effectiveness of fruit and vegetable intervention strategies}

The effects of 32 intervention strategies were synthesised from the 19 included reviews. There was evidence supporting the effectiveness of 19 of the 32 intervention strategies (Table 2). Among reviews reporting meta-analyses of 


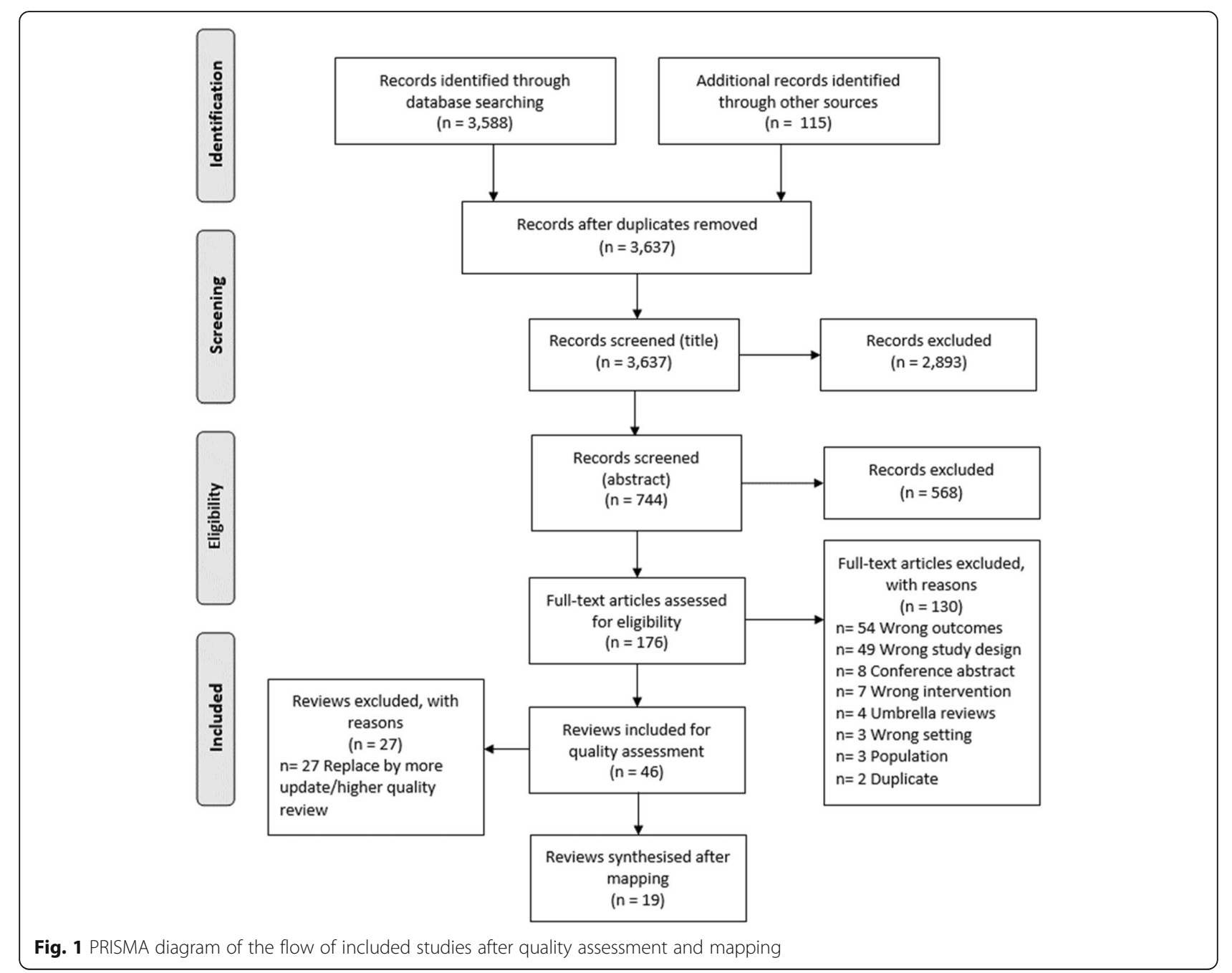

the effects on combined fruit and vegetable intake, effect sizes were largest for school-based strategies that provided free (or reduced price) fruits and vegetables, or increased their in-school availability $(+0.28$ serves); strategies that targeted child feeding practices in childcare services, and the home and family environment; and computer-based and SMS delivered intervention strategies. All of these intervention strategies improved intake by between 0.41 and 0.63 of a standard deviation (Additional file 3). Across reviews, intervention strategies that sought to increase the physical availability of fruits and vegetables in settings or communities appeared to be broadly effective, including those in school and workplace cafeterias, school gardens and domestic homebased agricultural settings.

Strategies that focused on nutrition awareness and education and skill development reported more mixed effects. For example, review evidence suggests that school-based nutrition education and curricula, mass media campaigns and nutrition counselling in primary care were effective in improving intake. More equivocal were review findings regarding the effects of nutrition education focused strategies delivered via childcare services, targeting parents and the home environment, or undertaken as part of individual and group-based community programs. Finally, although reductions or increases in the price of fruit and vegetable products were found to enhance (for price reductions of subsidies) or reduce (for price increases or taxes) fruit and vegetable intake, the long-term effects of other strategies that could be implemented within retail environments (e.g. choice architecture strategies) were mixed. Similarly, there was some evidence to support the introduction of mobile produce markets as a strategy to improve intake of fruits and vegetables among market users, but the introduction of new supermarkets (which offer both fresh and packaged foods) may reduce intake. The rest of this section outlines the effects of intervention strategies reported in the included reviews, grouped by setting where appropriate. 


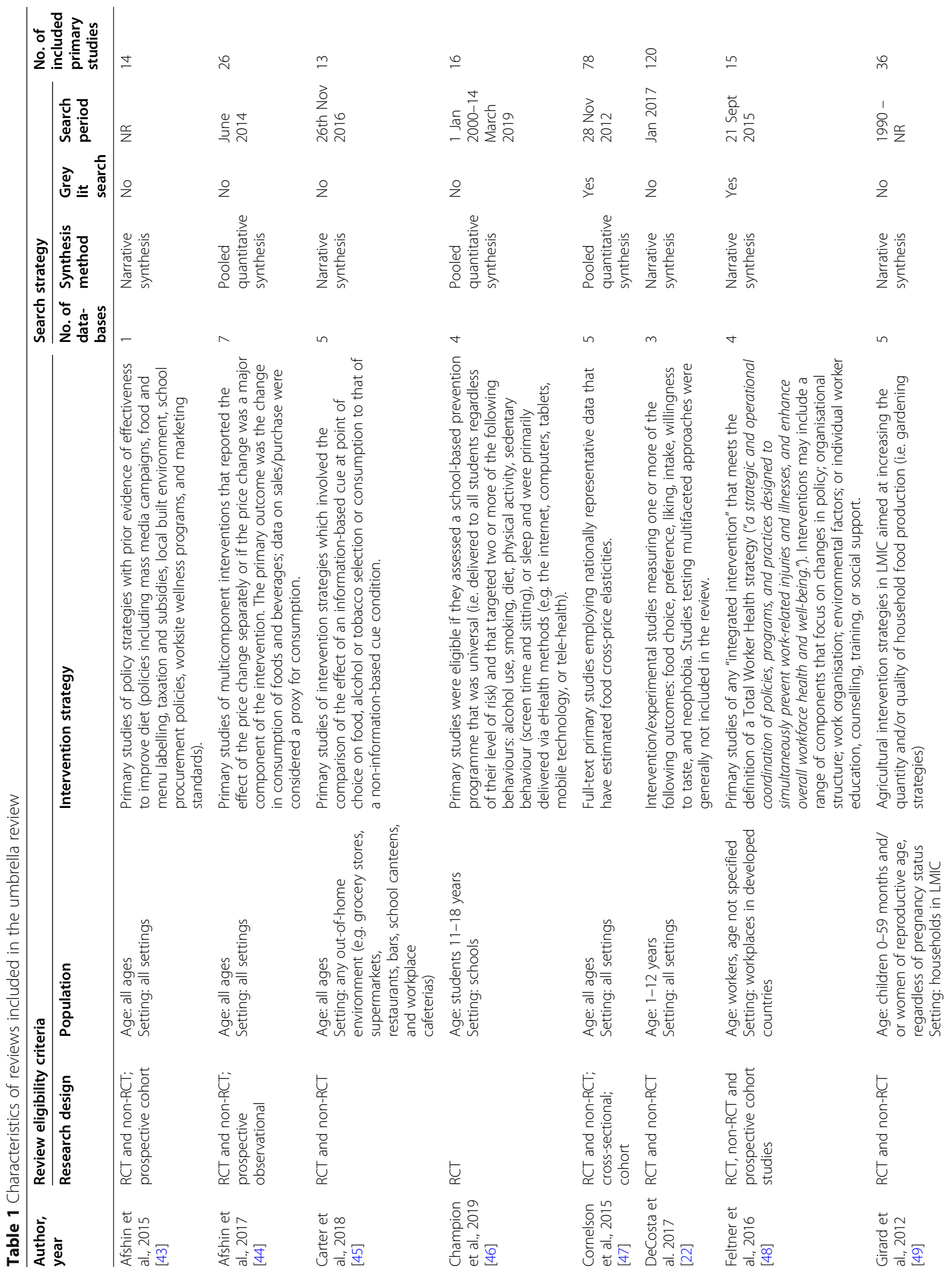




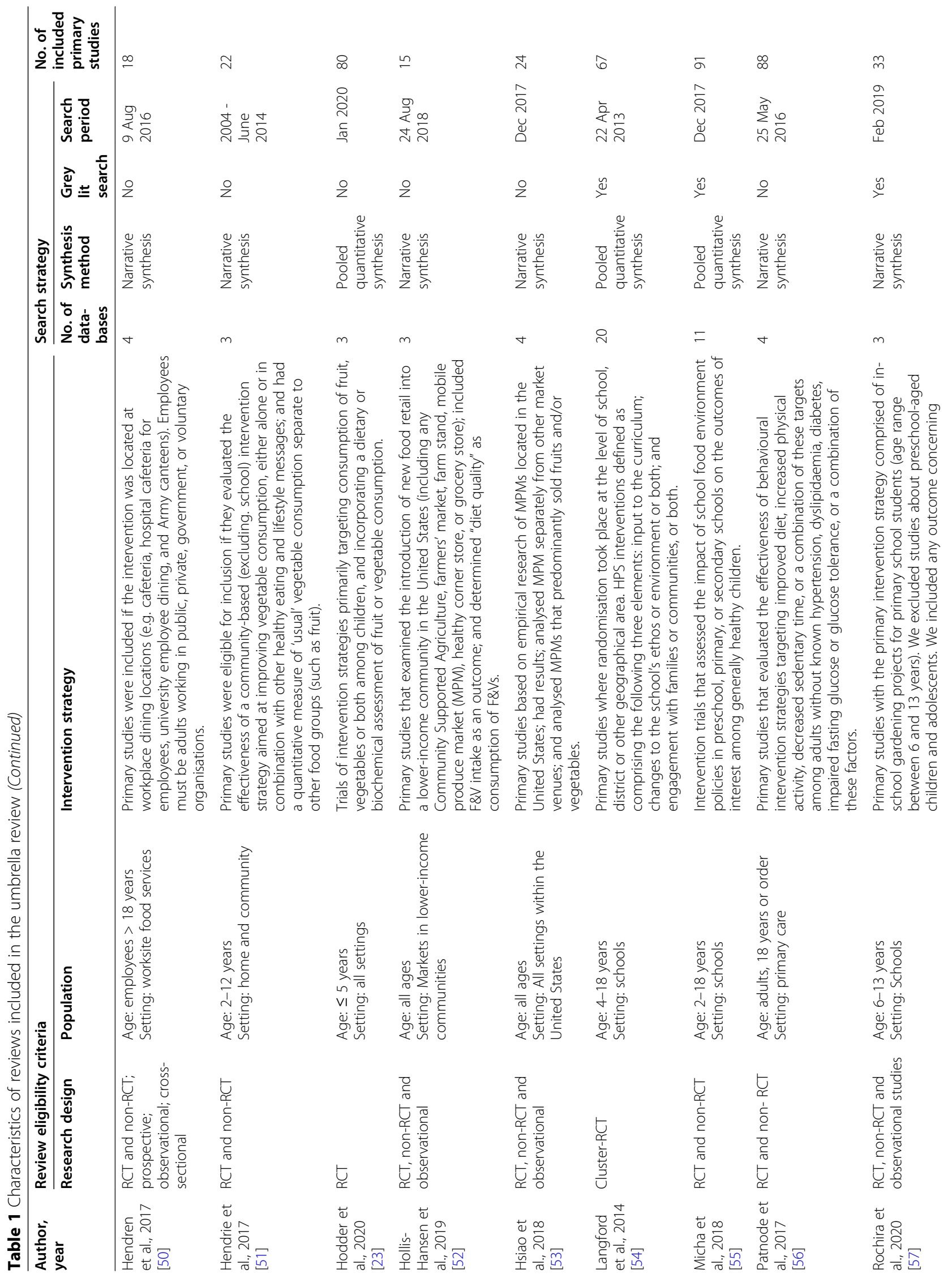




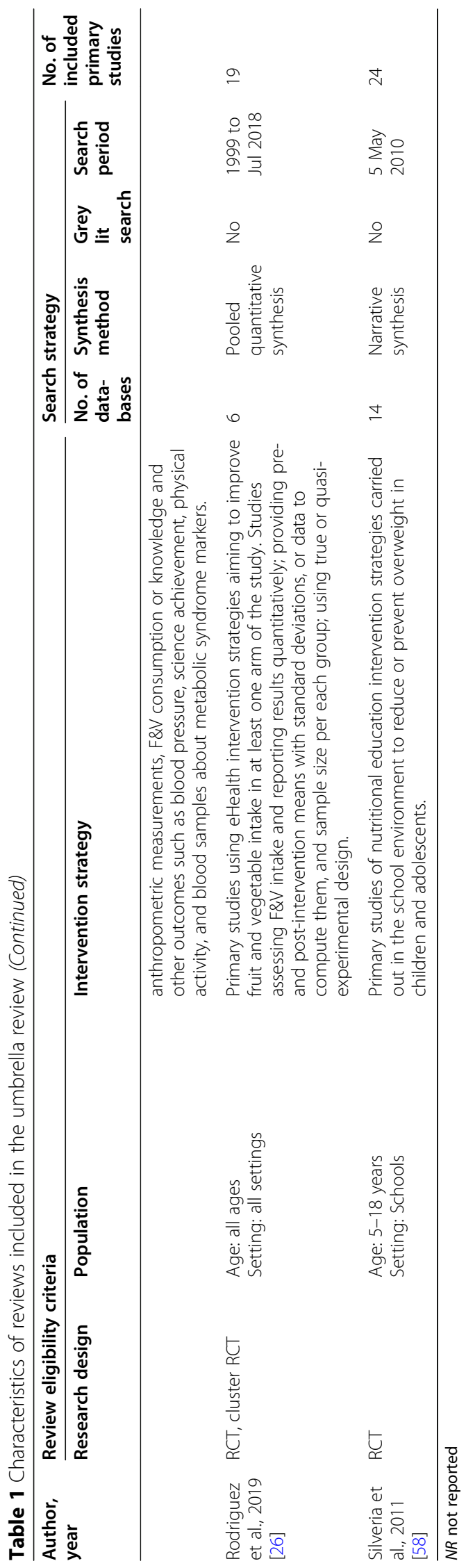




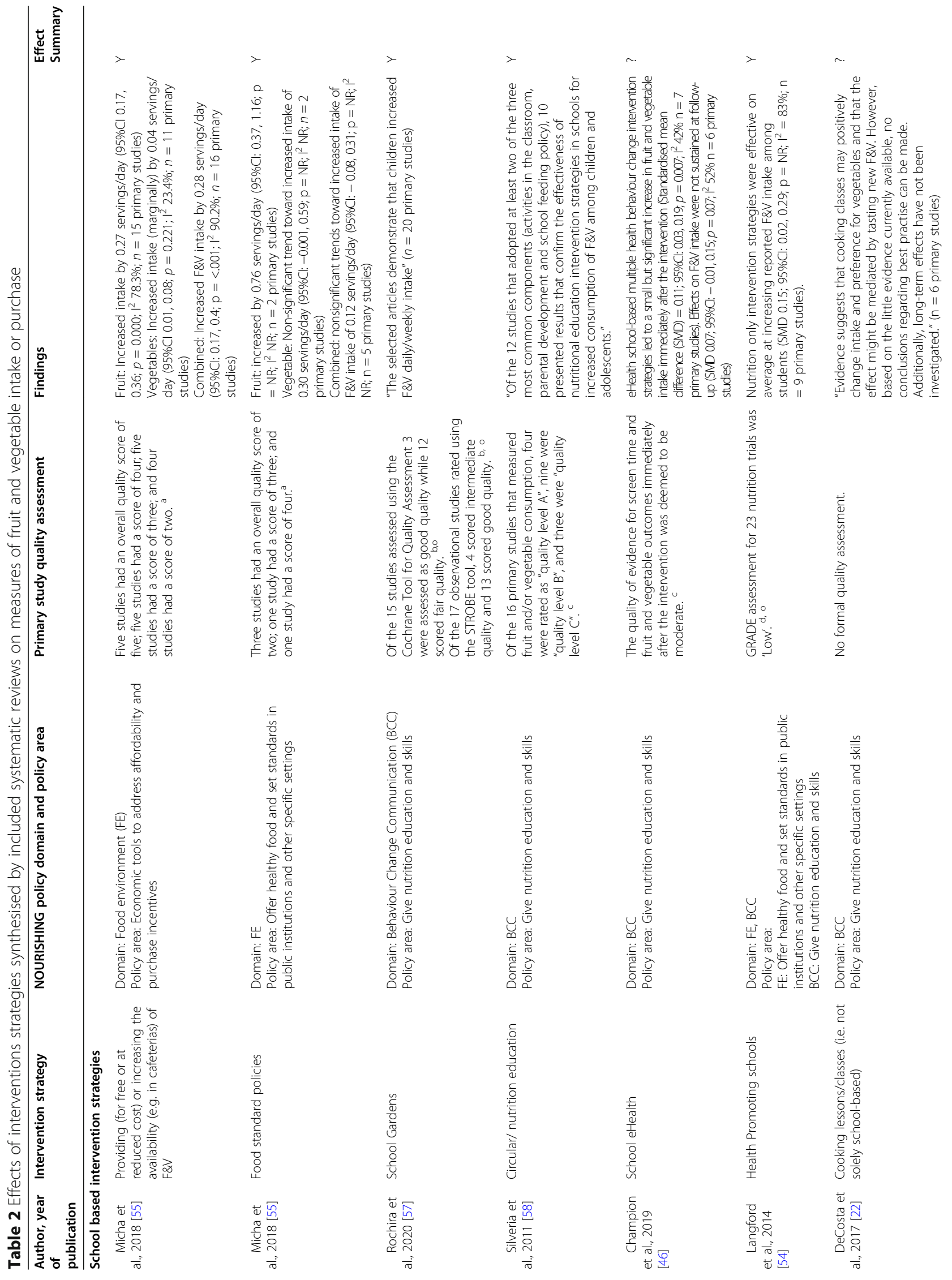




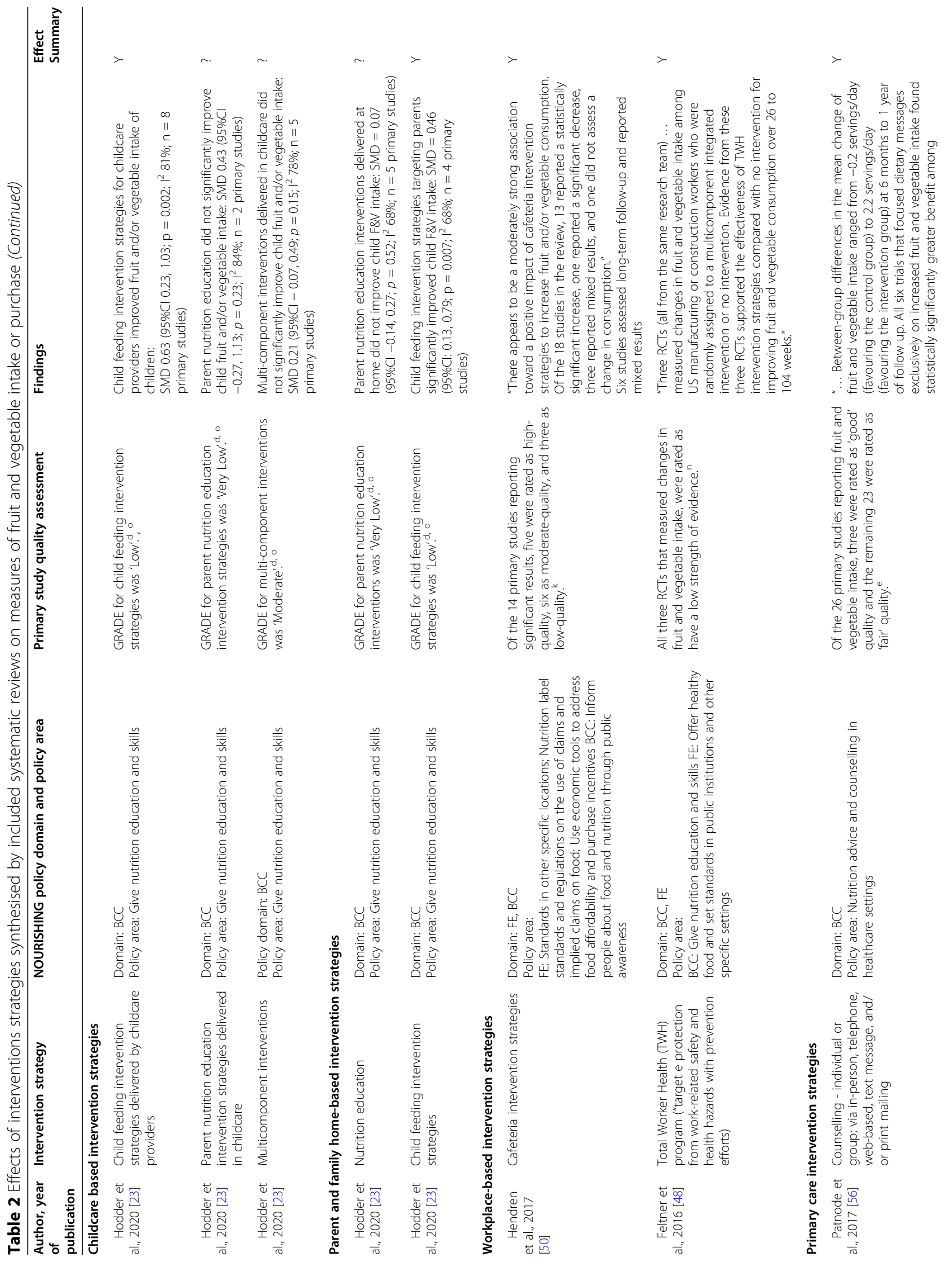




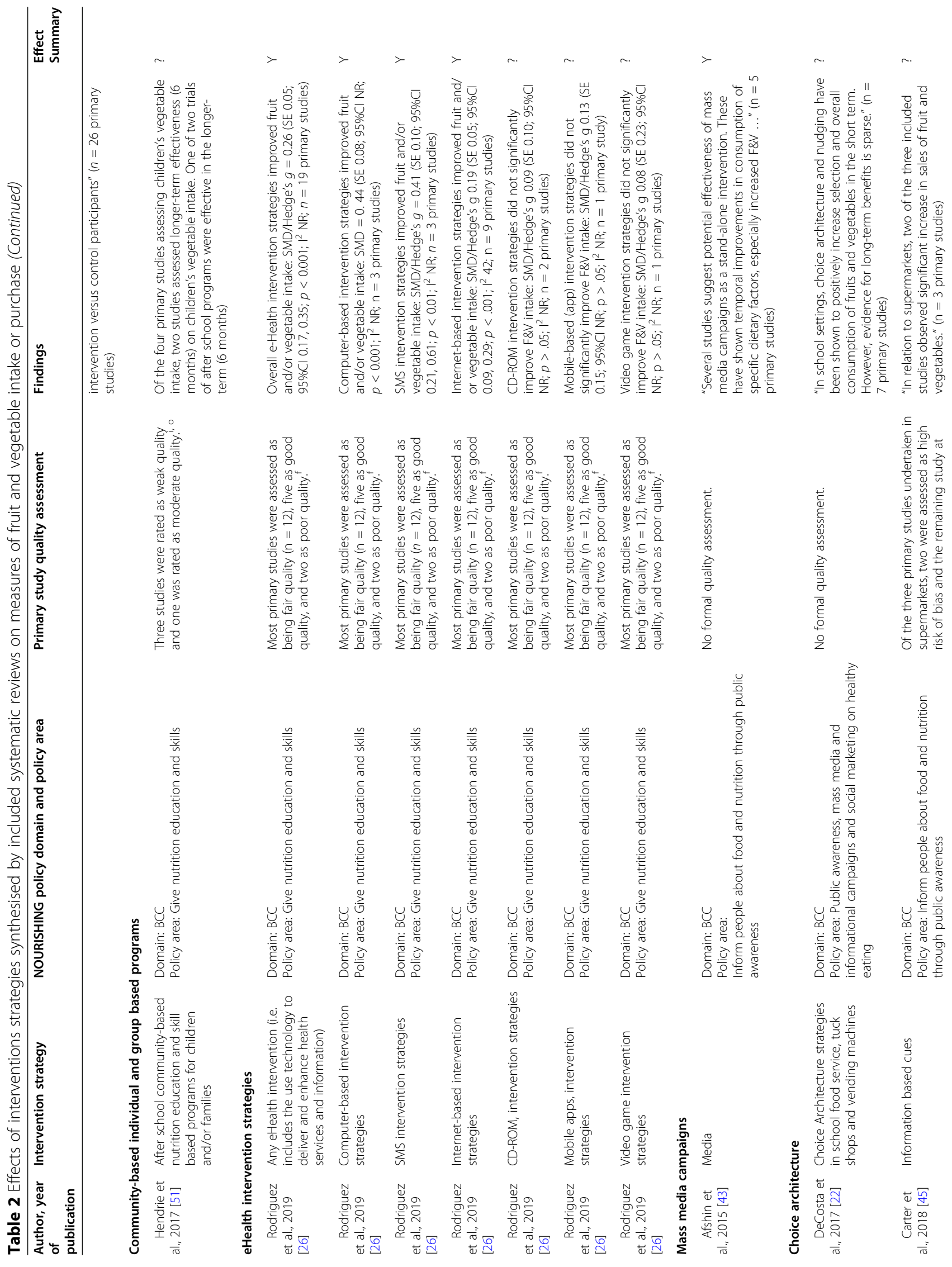




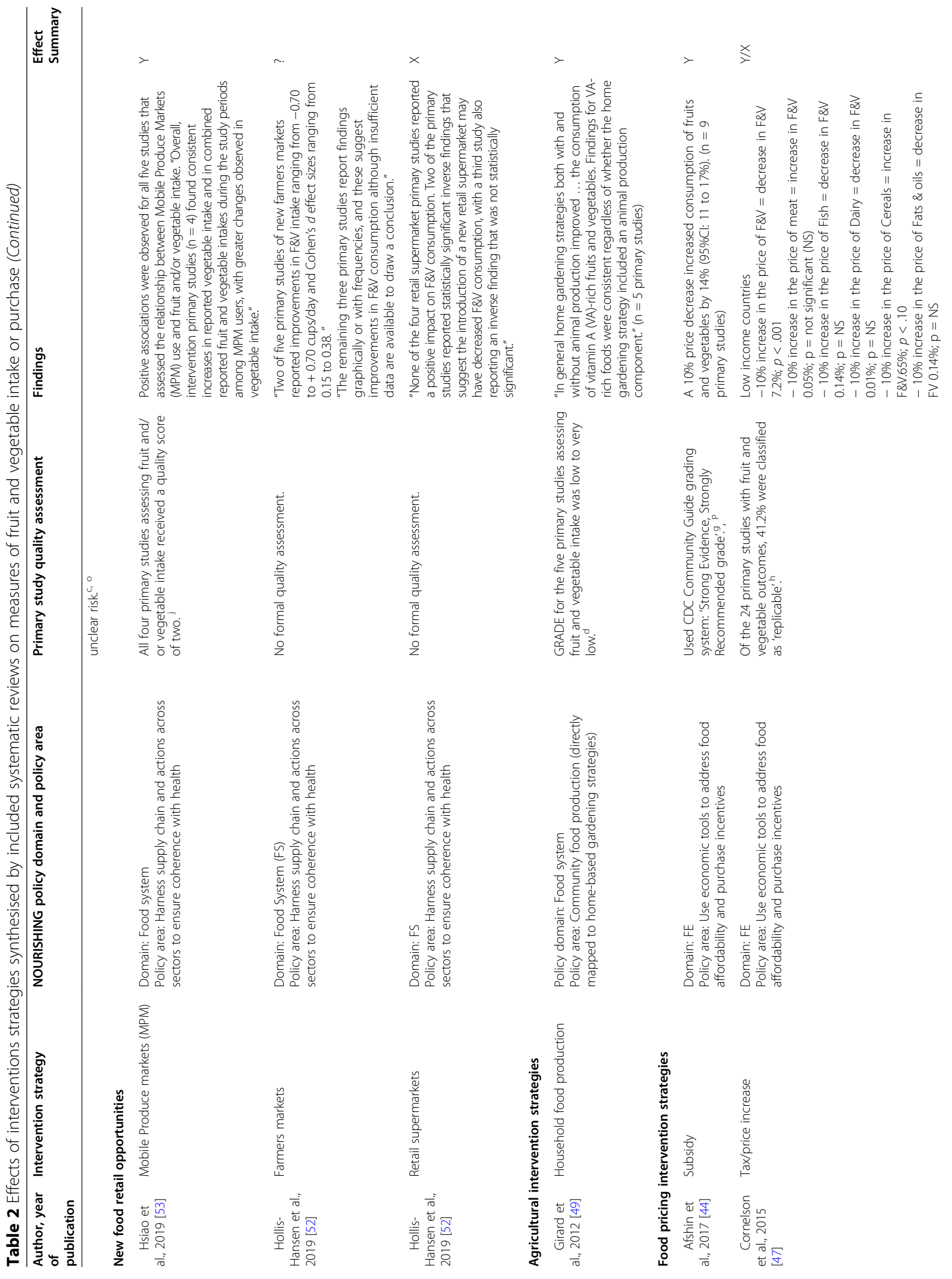




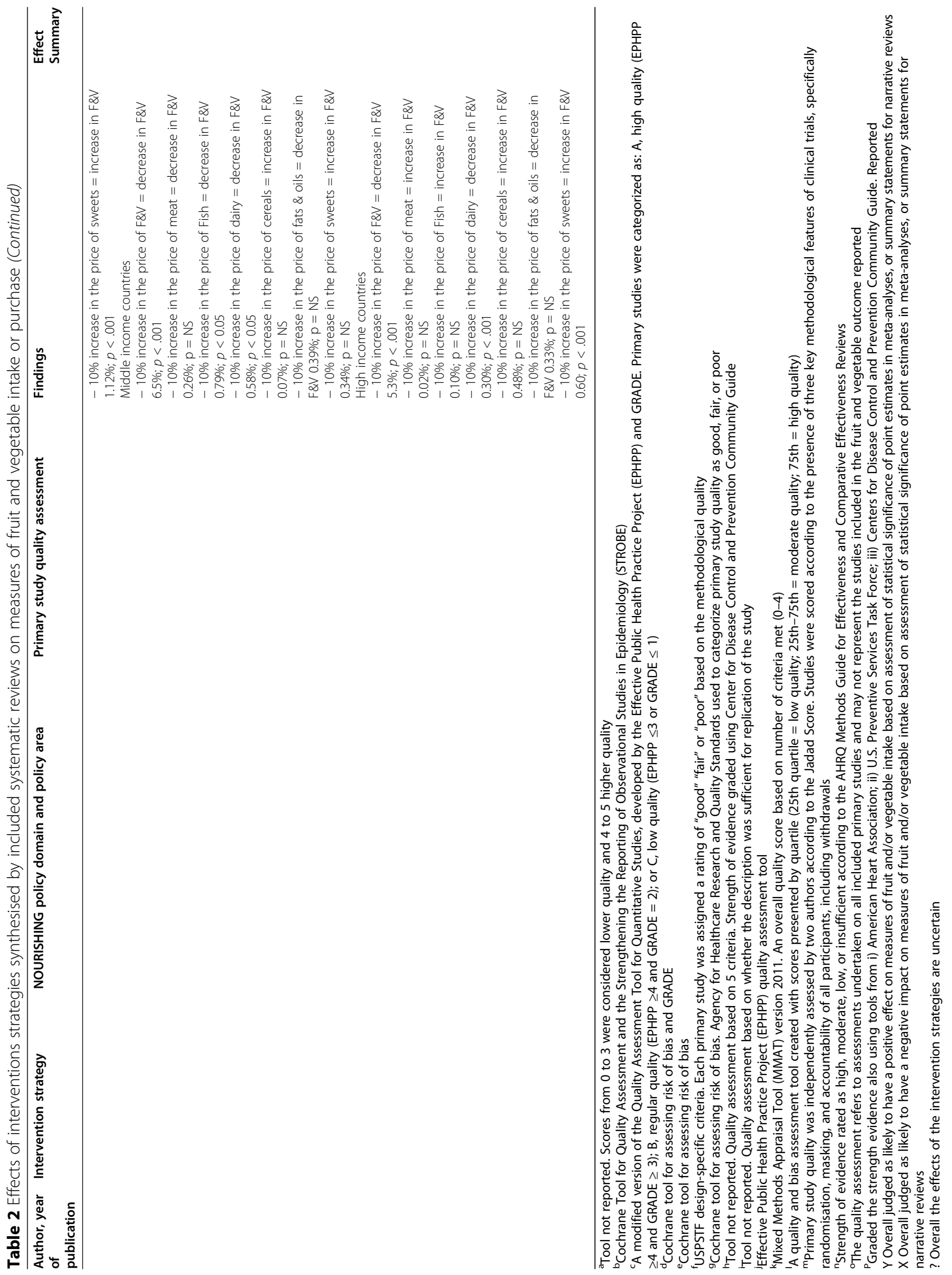




\section{Setting-based interventions}

School-based intervention strategies Six included systematic reviews reported the effects of seven school-based intervention strategies. In one of these reviews, which assessed the effects of cooking lessons, four of six primary studies were undertaken in the school setting (the other two occurred in the home) [22]. Five of the seven schoolbased intervention strategies were reported to be effective on at least one measure of fruit, vegetable, or combined fruit and vegetable intake, including providing free (or reduced price) fruits or vegetables, or increasing their within-school availability [55]; implementing food standard policies [55]; providing school gardens [57]; providing nutrition education and curricula [58]; and applying the Health Promoting Schools framework [54]. With the exception of nutrition education, effective intervention strategies were those targeting policy or modification of the school food environment. Among the reviews reporting meta-analyses, effect sizes were particularly high for food standard policies, which increased fruit intake by 0.76 serves/day (95\% confidence interval [CI]: 0.37, 1.16; $\mathrm{p}=$ not reported [NR]; $n=2$ primary studies) [55]. Reviews reporting on strategies focusing on communication and behaviour change via knowledge or skill acquisition - for example, cooking lessons [22] and school-based eHealth [46] - suggest that such strategies are effective immediately following the intervention, but in the long-term the effects are equivocal ( $n=6$ primary studies).

Childcare-based intervention strategies One review, reporting the effects of three childcare-based intervention strategies, was included. The review of randomised trials reported a significant pooled effect on child fruit and vegetable intake for child-feeding interventions delivered by childcare staff (standardized mean difference [SMD] $=0.63 ; 95 \% \mathrm{CI}: 0.23,1.03 ; p=0.002 ; n=8$ primary studies), but not for parent nutrition education intervention strategies delivered through childcare, or for multicomponent childcare intervention strategies.

Parent and family home-based intervention strategies One review, reporting the effects of two parent or family home-based intervention strategies, was included. A review of randomised trials found intervention strategies targeting child-feeding practices of parents (i.e. repeated food exposure) were effective in the short term $(<12$ months) on fruit and vegetable intake (SMD $=0.46 ; 95 \% \mathrm{CI}: 0.13,0.79 ; p=$ $0.007 ; n=4$ primary studies); however, parent nutrition education strategies were not effective [23].

Workplace-based intervention strategies Two reviews reported the effects of two workplace-based intervention strategies, the effects of which were mixed. In one review, strategies targeting workplace cafeterias (with or without nutrition education) [50] reported consistent improvements (from 13 of 18 primary studies) in fruit and vegetable intake post-intervention; however, evidence of effect at longer-term follow-ups (> 12 months) was equivocal. The other review examined intervention strategies to improve workplace health and safety integrated with health promotion interventions to advance worker well-being [48]. It cited evidence from three RCTs that demonstrated the effectiveness of such intervention strategies in improving fruit and vegetable intake over 26-104 weeks.

Primary-care intervention strategies One included review [56] examined the effects of primary care behavioural counselling interventions, either alone or as part of a larger multicomponent intervention on a range of behavioural risk factors for coronary heart diseases. The intervention strategies were delivered via a range of formats (e.g. face to face, telephone or web) and reported effect sizes ranging from -0.2 to 2.2 servings per day for fruits and vegetables ( $n=26$ primary studies). Heterogeneity of the primary studies precluded meta-analysis, but the review concluded that nutrition intervention strategies based in primary care generally resulted in small increases in fruit and vegetable intake.

Community-based individual and group-based programs One review examined community-based after-school programs [51]. It found that behavioural skills training and education with children and/or parents were effective in the short term (for vegetable intake only); however, the longer term effects ( $>6$ months) on measures of fruit or vegetable intake were mixed. The effects sizes on these measures ranged from an SMD of 0.18 to 0.25 ( $n=4$ primary studies).

\section{Non-setting-based interventions}

eHealth intervention strategies One review reported the effects of various eHealth intervention strategies [26]. An overall pooled analysis of all such strategies found they were effective in improving fruit and vegetable intake in adults and children (SMD $=0.26$; 95\%CI: 0.17, 0.35; $p<$ $0.001 ; \mathrm{I}^{2}$ not reported; $n=19$ primary studies). Subgroup analyses reported significant effects for computer-based, SMS and Internet-based intervention strategies, but not for those delivered via CD-ROM, mobile apps or video games.

Mass media intervention strategies A narrative synthesis of five primary studies of mass media intervention strategies targeting nutrition behaviours suggested the potential effectiveness of these as stand-alone approaches in improving the consumption of fruits and vegetables in adults and young people [43]. 
Choice architecture Two narrative reviews that examined the effects of choice architecture strategies reported mixed findings. One review did not disaggregate the effects of specific strategies, but found that evidence of the long-term effects of choice architecture intervention strategies (e.g. food signage, changes to food description, presentation and verbal prompts in cafeterias) was unclear overall ( $n=7$ primary studies) [22]. The other review found limited but supportive evidence from two of three primary studies that fruit and vegetable sales were improved by information-based cues in supermarket settings (excluding labels of nutritional content or nutritional values, and those delivered on TV or internet, or that were interactive) ( $n=3$ primary studies) [45].

New food retail opportunities Two reviews reported the effects of the introduction of three types of new food retail outlets. These reviews, which were restricted to primary studies undertaken in the US, [52, 53] suggested that intake of fruits and vegetables may be increased by the introduction of new retail opportunities that predominately provide access to fresh produce (e.g. fruits and vegetables), but not by opportunities that increase access to a broader range of fresh and packaged foods. Specifically, it was suggested that mobile produce markets increase fruit and vegetable intake among market users $(n=4$ primary studies), [53] farmers markets introduced in lower income communities have mixed effects $(-0.70-0.70$ cups per day), [52] and new food retail markets (supermarkets) may have a detrimental impact on fruit and vegetable intake (4 primary studies) [52].

Agricultural intervention strategies One review [49] of randomised and non-randomised trials in LMIC examined the effects of household food production intervention strategies on the nutrition and health outcomes of women and children ( $n=5$ primary studies). It concluded that household gardens, with or without an animal production component, improved intake of fruits and vegetables rich in vitamin A rich.

Food pricing intervention strategies Two reviews reported the effects of two fiscal intervention strategies: price reductions and price increases. One review [44] pooled data from nine interventional and prospective cohort studies, and found that a $10 \%$ decrease (or subsidy) in the price of fruits and vegetables increased their consumption by $14 \%$ (95\%CI: 11 to $17 \%)$ ( $n=9$ primary studies). The other review [47] found that a $10 \%$ increase (e.g. tax) was associated with reductions in consumption of fruits and vegetables of 7.2, 6.5 and $5.3 \%$ in low-, medium- and high-income countries, respectively $(n=$ 24 primary studies). Price increases on other food types, such as sweets, were found to have a marginal positive impact on consumption of fruits and vegetables [47].

\section{Review evidence gaps}

Table 3 illustrates how the intervention strategies synthesised in the included systematic reviews align with policy action areas suggested in the WCRF NOURISHING framework (represented by the shaded cells). The intervention strategies could be mapped across all three broad framework domains (food environment, food system and behaviour change communication) and across seven of the 10 broad policy areas. However, they covered just 14 of the 65 specific sub-policy areas in the framework (see Table 3); thus, systematic review evidence is not available for most of the recommended sub-policy areas. Intervention strategies were mapped most frequently to sub-policy areas of the behaviour change and communication domain of the framework. Very little review evidence described the effects of intervention strategies in the food system domain. Nonetheless, the included reviews provided evidence supporting the effectiveness of intervention strategies in most of the sub-policy areas for which reviews could be mapped.

All the intervention strategies synthesised by the included reviews that mapped to the 'food environment' domain were reportedly effective in improving fruit and vegetable intake, including those within the policy areas of 'Nutrition label standards and regulations' and 'Offering healthy food and set standards in public institutions and other settings', such as schemes to increase the availability of fruits and vegetables in schools. There was also direct evidence from systematic reviews regarding the impact of interventions in the policy area of 'Use of economic tools to address food affordability and purchase incentives'. This evidence supported sub-policy areas such as the use of food taxes [47] on foods such as sweets (to reduce intake), and targeted subsidies for fruits and vegetables [43].

In contrast to intervention strategies mapped to the food environment domain, the strategies mapped to the food system and behaviour change communication domain did not always have a beneficial effect. Within the 'food system domain', evidence from systematic reviews could be mapped directly to just one sub-policy area 'Community food production' - for which reviews found that home gardens in LMIC improved fruit and vegetable consumption, whereas evidence of the benefits of introducing fresh-produce markets was less certain [49].

Within the 'Behaviour change communication domain', direct evidence of a beneficial effect was identified within the policy areas of 'Informing people about food and nutrition through public awareness' (specifically, the use of mass media campaigns) [43] and 'nutrition advice and counselling in healthcare settings' (specifically, nutrition counselling in primary care) [56]. Within the policy area 
of 'Give nutrition education and skills', systematic review evidence could be directly mapped to nutrition education on curricula, [58] initiatives to train school children on growing food [57] and workplace health schemes, [48] each of which was reported as being effective in improving fruit and vegetable intake. However, within this policy area, evidence of the beneficial effects of community-based nutrition education [51] and cooking skills [22] was equivocal.

\section{Discussion}

This umbrella review sought to consolidate the global evidence base from systematic reviews regarding the effectiveness of intervention strategies to improve fruit and vegetable intake. It identified several strategies with evidence of a beneficial impact, mapped to the WCRF NOURISHING framework, including those undertaken in community settings (e.g. schools, childcare services and workplaces), eHealth and mass media, household food production strategies, and fruit and vegetable subsidies. The findings indicate that policy-makers and practitioners have a range of effective options to improve population level intake of fruits and vegetables. Nonetheless, the evidence on the effects of intervention strategies represented only a fraction of the options suggested by comprehensive nutrition frameworks; thus, many recommended nutrition actions have not been the subject of systematic synthesis to determine their specific impact on fruit and vegetable intake.

Intervention strategies for which systematic reviews reported an effect were predominately focused on the food environment and behaviour change communication domains of the WCRF NOURISHING framework, and most of these reviews reported the effects of setting-based interventions (particularly schools). Encouragingly, schoolbased fruit and vegetable intervention strategies are frequently undertaken by governments, and this umbrella review provides further evidence to support investment in such initiatives. However, the included reviews did not assess the impacts of most of the framework sub-category areas, and none of them reported the effects of intervention strategies within the broad categories of 'Restrictions on food advertising and commercial promotion' or 'Improving the quality of the whole food supply'. Strategies such as nutrition labelling and food reformulation can improve energy or macronutrient intake, $[59,60]$ and are often recommended as pillars of public health approaches to prevent chronic diseases [61]. Although these types of strategy may not directly target fruit and vegetable intake, they could conceivably increase it. The conduct of primary studies and systematic reviews to address these identified evidence gaps is warranted, to provide further evidence to justify the application of such strategies.

Pricing strategies have been suggested as a powerful determinant of dietary behaviour. Their use to improve public health nutrition has been recommended by the
UN General Assembly at a High-Level Meeting on NonCommunicable Diseases [62]. Indeed, countries such as Denmark, Hungary, Mexico and the US have introduced fiscal strategies targeting food and drink items [63]. The findings of this review provide evidence to support the influence of changes in the relative price of fruits and vegetables on their intake, particularly in LMIC. Specifically, reducing the cost of fruits and vegetables by $10 \%$ (through the introduction of subsidies) can increase intake by $14 \%$, while price increases of the same magnitude will reduce consumption by $5-7 \%$. Taxes on other food products (e.g. sweets) were also found to increase fruit and vegetable intake, albeit marginally, suggesting that raising the price of unhealthy foods may contribute to healthier diets more broadly [47]. Introducing both subsidies and taxes may represent a particularly potent tool for increasing fruit and vegetable consumption. Modelling undertaken in Australia, for example, suggests that such an approach can yield significant health gains, and cost savings from a health system perspective [63]. Fiscal measures, however, need to be carefully designed to ensure that they do not exacerbate inequities, given their disproportionate impact on the most financially disadvantaged population groups.

Access to fruits and vegetables is a critical determinant of intake [64]. This umbrella review confirmed that schoolbased strategies to improve the availability to fruit and vegetable products are an effective way to increase child intake of these products. More broadly, research suggests that improving the availability of healthy foods within schools, [55] workplace food services or cafeterias, [50] or other food outlets [65] is associated with greater intake of these foods. However, improving availability may be particularly challenging in countries where the supply of fruits and vegetables is limited [13]. Within such settings, choice architecture, coupled with strategies to increase the accessibility and palatability of fruits and vegetables, represent promising avenues to further increase intake and reduce wastage, although more research is required to confirm the effects of these intervention strategies in the longer term [22].

Interestingly, the umbrella review found mixed effects reported from efforts to improve the physical availability of fruits and vegetables through new food retail outlets, such as supermarket grocery stores, mobile produce markets and farmers markets. Farmers and mobile produce markets were suggested to be beneficial in improving fruit and vegetable intake because they provide access to fresh produce, including fruits and vegetables. In contrast, the introduction of new retail supermarkets appeared to have a detrimental effect on fruit and vegetable intake. Also supermarkets may increase the availability of fruits and vegetables, they also increase access to less healthy foods and exposure to unhealthy food marketing, which may explain, in part, these apparently contradictory findings. 
Table 3 Evidence from systematic reviews synthesised in the umbrella review mapped to the NOURISHING Framework

\begin{tabular}{|c|c|c|c|c|c|c|c|c|c|c|}
\hline 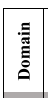 & \multicolumn{6}{|c|}{ Food Environment(22, 47, 51, 54, 56-58) } & \multirow{2}{*}{\begin{tabular}{|l|} 
Food System(43- \\
\multicolumn{4}{|c|}{$45)$} \\
\\
\\
H- Harness food \\
supply chain and \\
actions across \\
sectors to ensure \\
coherence with \\
health \\
DM(43-45)
\end{tabular}} & \multicolumn{3}{|c|}{$\begin{array}{c}\text { Behaviour Change Communication }(23,26 \text {, } \\
46,48-50,52,53,56,58)\end{array}$} \\
\hline 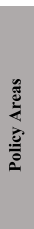 & \begin{tabular}{|l|} 
N-Nutrition \\
label \\
standards and \\
regulations on \\
the use of \\
claims and \\
implied \\
claims on \\
food \\
IM(58)
\end{tabular} & \begin{tabular}{|l|} 
O- Offer \\
healthy \\
food and set \\
standards \\
in public \\
institutions \\
and other \\
specific \\
settings \\
DM(51) \\
IM(47, 58$)$
\end{tabular} & \begin{tabular}{|l} 
U -Use \\
economic \\
tools to \\
address \\
food \\
affordability \\
and \\
purchase \\
incentives \\
DM(54,57, \\
$58)$
\end{tabular} & \begin{tabular}{|l} 
R- Restrict \\
food \\
advertising \\
and other \\
forms of \\
commercial \\
promotion
\end{tabular} & $\begin{array}{l}\text { I - Improve } \\
\text { nutritional } \\
\text { quality of } \\
\text { the whole } \\
\text { food supply }\end{array}$ & \begin{tabular}{|l} 
S-Set \\
incentives and \\
rules to create \\
a healthy retail \\
and food \\
service \\
environment
\end{tabular} & & $\begin{array}{l}\text { I - Inform } \\
\text { people about } \\
\text { food and } \\
\text { nutrition } \\
\text { through } \\
\text { public } \\
\text { awareness } \\
\text { DM(22,53) } \\
\text { IM(58) }\end{array}$ & \begin{tabular}{|l|}
$\mathbf{N}-$ \\
Nutrition \\
advice and \\
counselling \\
in \\
healthcare \\
settings \\
DM(52)
\end{tabular} & $\begin{array}{l}\text { G- Give } \\
\text { nutrition } \\
\text { education } \\
\text { and skills } \\
\text { DM(46, 48, } 55, \\
56(2), 4,6) \\
\operatorname{IM(22,47,66)} \\
0\end{array}$ \\
\hline \multirow[t]{10}{*}{ 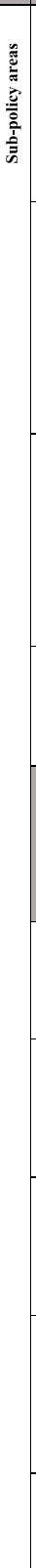 } & \begin{tabular}{|l|} 
Mandatory \\
nutrient lists \\
on packaged \\
food
\end{tabular} & \begin{tabular}{|l|}
$\begin{array}{l}\text { Fruit \& } \\
\text { regetable } \\
\text { initiatives in } \\
\text { schools } \\
\text { DM(51) }\end{array}$ \\
\end{tabular} & \begin{tabular}{|l|} 
Health- \\
related food \\
taxes \\
DM(57) \\
\end{tabular} & \begin{tabular}{|l|} 
Mandatory \\
regulation of \\
broadcast food \\
advertising to \\
children
\end{tabular} & \begin{tabular}{|l|}
$\begin{array}{l}\text { Voluntary } \\
\text { reformulation } \\
\text { of food } \\
\text { products }\end{array}$ \\
\end{tabular} & \begin{tabular}{|l|} 
Incentives and \\
rules for stores \\
to locate in \\
under-served \\
neighbourhoods
\end{tabular} & $\begin{array}{l}\text { Working with food } \\
\text { suppliers to provide } \\
\text { healthier } \\
\text { ingredients }\end{array}$ & $\begin{array}{l}\text { Development } \\
\text { and } \\
\text { communication } \\
\text { of food-based } \\
\text { dietary } \\
\text { guidelines }\end{array}$ & \begin{tabular}{|l|} 
Guidelines \\
and \\
programmes \\
to provide \\
support in \\
primary care \\
to people \\
who are \\
overweight \\
and obese \\
\end{tabular} & 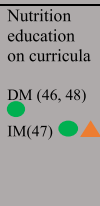 \\
\hline & \begin{tabular}{|l|} 
Trans fats \\
included in \\
mandatory \\
nutrient labels \\
\end{tabular} & \begin{tabular}{|l|} 
Mandatory \\
standards \\
for food \\
available in \\
schools \\
including \\
restrictions \\
on \\
unhealthy \\
food \\
DM(51) \\
IM(47)
\end{tabular} & \begin{tabular}{|l|} 
Voluntary \\
health- \\
related food \\
taxes
\end{tabular} & \begin{tabular}{|l|} 
Mandatory \\
regulation of \\
food advertising \\
on non- \\
broadcast \\
communications \\
channels
\end{tabular} & \begin{tabular}{|l|} 
Voluntary \\
commitments \\
to reduce \\
portion sizes \\
\end{tabular} & \begin{tabular}{|l|} 
Initiatives to \\
increase the \\
availability of \\
healthier food \\
in stores and \\
food service \\
outlets
\end{tabular} & $\begin{array}{l}\text { Nutrition standards } \\
\text { for public } \\
\text { procurement }\end{array}$ & $\begin{array}{l}\text { Development } \\
\text { and } \\
\text { communication } \\
\text { of guidelines } \\
\text { for specific } \\
\text { food groups }\end{array}$ & \begin{tabular}{|l|}
$\begin{array}{l}\text { Nutrition } \\
\text { counselling } \\
\text { in primary } \\
\text { care }\end{array}$ \\
$\mathrm{DM}(52)$
\end{tabular} & $\begin{array}{l}\text { Community- } \\
\text { based } \\
\text { nutrition } \\
\text { eduction } \\
\text { IM(50) }\end{array}$ \\
\hline & \begin{tabular}{|l|} 
Clearly visible \\
"interpretative" \\
labels and \\
warning labels
\end{tabular} & \begin{tabular}{|l|} 
Mandatory \\
standards \\
for food \\
available in \\
schools and \\
in their \\
immediate \\
vicinity \\
\end{tabular} & \begin{tabular}{|l} 
Increasing \\
import tariffs \\
on specified \\
"unhealthy" \\
food
\end{tabular} & \begin{tabular}{|l|} 
Mandatory \\
regulation of \\
food advertising \\
through any \\
medium
\end{tabular} & $\begin{array}{l}\text { Mandatory } \\
\text { limits on } \\
\text { level of salt } \\
\text { in food } \\
\text { products }\end{array}$ & \begin{tabular}{|l|} 
Incentives and \\
rules to reduce \\
trans fat in food \\
service outlets
\end{tabular} & $\begin{array}{l}\text { Public procurement } \\
\text { through "short" } \\
\text { chains (eg local } \\
\text { farmers) }\end{array}$ & \begin{tabular}{|l|} 
Public \\
awareness, \\
mass media \\
and \\
informational \\
campaigns and \\
social \\
marking on \\
healthy eating \\
DMM(2.,53)
\end{tabular} & \begin{tabular}{|l|}
$\begin{array}{l}\text { Training for } \\
\text { health } \\
\text { professionals }\end{array}$ \\
\end{tabular} & $\begin{array}{l}\text { Cooking } \\
\text { skills } \\
\text { IM(22) }\end{array}$ \\
\hline & \begin{tabular}{|l|}
$\begin{array}{l}\text { On-shelf } \\
\text { labelling }\end{array}$ \\
\end{tabular} & \begin{tabular}{|l|} 
Voluntary \\
guidelines \\
for food \\
available in \\
schools \\
\end{tabular} & \begin{tabular}{|l} 
Lowering \\
import tariffs \\
on specified \\
"healthy" \\
food
\end{tabular} & \begin{tabular}{|l|} 
Mandatory \\
regulation of \\
specific \\
marketing \\
techniques
\end{tabular} & 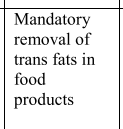 & \begin{tabular}{|l|} 
Incentives and \\
rules to offer \\
healthy food \\
options as a \\
default in food \\
service outlets
\end{tabular} & $\begin{array}{l}\begin{array}{l}\text { Supply chain } \\
\text { incentives for food } \\
\text { production }\end{array} \\
\end{array}$ & $\begin{array}{l}\text { Public } \\
\text { awareness } \\
\text { campaigns } \\
\text { specific to fruit } \\
\text { and vegetables }\end{array}$ & & $\begin{array}{l}\text { Initiatives to } \\
\text { train school } \\
\text { children on } \\
\text { growing } \\
\text { food } \\
\text { DM(55) }\end{array}$ \\
\hline & \begin{tabular}{|l|} 
Calorie and \\
nutrient \\
labelling on \\
menus and \\
displays in \\
out-of home \\
venues \\
IMrs8
\end{tabular} & \begin{tabular}{|l|} 
Bans \\
specific to \\
vending \\
machines in \\
schools \\
\end{tabular} & $\begin{array}{l}\text { Targeted } \\
\text { subsidies for } \\
\text { healthy food } \\
\text { DM(54) } \\
\text { IM(58) }\end{array}$ & \begin{tabular}{|l|} 
Mandatory \\
regulation of \\
marketing of \\
specific food \\
items and \\
beverages \\
\end{tabular} & \begin{tabular}{|l|} 
Limits on the \\
availability \\
of high-fat \\
meat \\
products
\end{tabular} & \begin{tabular}{|l|} 
Incentives and \\
rules to restrict \\
sugar- \\
sweetened \\
beverage \\
consumption \\
\end{tabular} & $\begin{array}{l}\text { Supporting urban } \\
\text { agriculture in } \\
\text { health and planning } \\
\text { policies }\end{array}$ & \begin{tabular}{|l|} 
Public \\
awareness \\
campaigns \\
concening \\
specific \\
unhealthy food \\
and beverages*
\end{tabular} & & 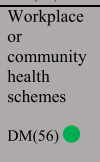 \\
\hline & \begin{tabular}{|l|} 
Warning labels \\
on menus and \\
displays in \\
out-of-home \\
venues \\
\end{tabular} & \begin{tabular}{|l|} 
Standards in \\
social \\
support \\
programmes
\end{tabular} & & \begin{tabular}{|l|} 
Mandatory \\
regulation of \\
food marketing \\
in schools
\end{tabular} & \begin{tabular}{|l|} 
Limits on the \\
availability \\
of high-sugar \\
food \\
products and \\
beverages
\end{tabular} & \begin{tabular}{|l|} 
Incentives and \\
rules to reduce \\
salt in food \\
service outlets
\end{tabular} & $\begin{array}{l}\text { Community food } \\
\text { production } \\
\text { DM(43-45) }\end{array}$ & $\begin{array}{l}\text { Public } \\
\text { awareness } \\
\text { campaigns } \\
\text { concerning salt }\end{array}$ & & $\begin{array}{l}\text { Training for } \\
\text { caterers and } \\
\text { food service } \\
\text { providers }\end{array}$ \\
\hline & \begin{tabular}{|l|} 
Rules on \\
nutrient claims \\
(ie nutrient \\
content and \\
nutrient \\
comparative \\
claims)
\end{tabular} & \begin{tabular}{|l|} 
Standards in \\
other \\
specific \\
locations \\
IM(58)
\end{tabular} & & \begin{tabular}{|l} 
Mandatory \\
requirement that \\
advertisements \\
must carry a \\
health message \\
or warning
\end{tabular} & & \begin{tabular}{|l|} 
Planning \\
restrictions on \\
food outlets
\end{tabular} & 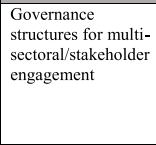 & & & \\
\hline & \begin{tabular}{|l|} 
Rules on \\
health claims \\
ie nutrient \\
function and \\
disease risk \\
reduction \\
claims) \\
\end{tabular} & & & \begin{tabular}{|l|} 
Voluntary \\
regulation of \\
food advertising \\
on non- \\
broadcast \\
communications \\
channels
\end{tabular} & & & & & & \\
\hline & & & & \begin{tabular}{|l|} 
Government \\
engage with \\
industryy to \\
develop self- \\
regulation to \\
restrict food \\
marketing to \\
children \\
\end{tabular} & & & & & & \\
\hline & & & & \begin{tabular}{|l|}
$\begin{array}{l}\text { Government } \\
\text { support } \\
\text { volutary } \\
\text { pledges by } \\
\text { industry }\end{array}$ \\
\end{tabular} & & & & & & \\
\hline
\end{tabular}


The findings of the review should be considered in the context of several limitations. First, as an umbrella review, the study was restricted to the analyses reported in the included reviews, and did not reexamine or analyse primary studies. Thus, the identified gaps in the evidence base for some intervention strategies may reflect the lack of systematic reviews of these strategies rather than the absence of primary studies. Second, it is likely that there is some duplication of primary studies in the syntheses of the effects of intervention strategies reported by the included reviews, particularly among reviews reporting strategies conducted in the same setting (e.g. schools). Third, review inclusion criteria were restricted to prospective evaluations of studies reporting measures of fruit and vegetable intake (or purchasing). Some intervention strategies (e.g. those targeting modification of the food supply) may not be readily amendable to evaluation using conventional prospective designs, or their effects may be more appropriately assessed using other metrics (e.g. sales volume or availability) that can be correlated with population level intakes. Including broader study design and outcome measures in the eligibility criteria of the umbrella review may have identified other relevant reviews. Anecdotally, however, such systematic reviews were not apparent during screening for this review. The included systematic reviews also largely examined intervention strategies that were more commonly implemented in high-income countries, and most of the primary studies included in these systematic reviews were from high-income nations, reflecting the available published literature. Hence, the global applicability of the findings of some strategies may be limited, underscoring the need for both primary studies and reviews specific to intervention strategies undertaken in LMIC.

Notwithstanding its limitations, the review identifies a range of options for health policy makers and practitioners interested in improving the health and wellbeing of communities through strategies to improve fruit and vegetable intake. In particular, the review provides evidence of a beneficial effect for setting-based and fiscal approaches. Many intervention strategies based on the food system (e.g. those targeting agricultural production practices or the supply chain, or more macro-level intervention strategies such as international trade agreements or climate change policies) could have a profound impact on population-level fruit and vegetable consumption; however, their effects have not been reported in systematic reviews. The conduct of primary studies assessing the impact of such approaches and their inclusion in systematic reviews would better support appraisal of their benefits, which in turn would help to strengthen national and international public health nutrition efforts.

\section{Supplementary Information}

The online version contains supplementary material available at https://doi. org/10.1186/s12966-020-01046-y.

Additional file 1. Search strategy (contains details of the search terms used in database searches).

Additional file 2. Quality assessment of included reviews (contains details on the quality assessments for each review).

Additional file 3. Pooled effects of intervention strategies to improve fruit and vegetable uptake.

\begin{abstract}
Abbreviations
CA: critical appraisal; Cl: confidence interval; FAO: Food and Agriculture Organization of the United Nations; GRADE: Grading of Recommendations, Assessment, Development and Evaluations; LMIC: low-and middle-income countries; NCDs: non-communicable diseases; PRIOR: Preferred Reporting Items for Overviews of Reviews; PRISMA: Preferred Reporting Items for Systematic Reviews and Meta-Analyses; RCT: randomised controlled trial; SMD: standardised mean difference; UN: United Nations; US: United States; WCRF: World Cancer Research Fund International; WHO: World Health Organization
\end{abstract}

\section{Acknowledgements}

The authors would like to acknowledge Debbie Booth for her help developing the search strategy and Miranda Cumpston for her assistance with the forest plot. We would also like to acknowledge the contribution of experts from the Food and Agricultural Organization of the United Nations, World Health Organization and other institutions who provided expert review of the manuscript; and the expert comments of the peer reviewers and Editors of IJBNPA whose constructive input and persistence enhanced the quality of the manuscript. Finally, we appreciate the copy-editing services provided by Hilary Cadman.

\section{Authors' contributions}

LW, SG, SB, LSV and AM developed the initial manuscript concept and design. $\mathrm{LW}, \mathrm{CB}$ and $\mathrm{CL}$ were responsible for screening and data extraction. LW led analysis and manuscript write-up. SMC and HB completed quality assessments of included reviews. AM, and LSV were responsible for screening and data extraction. All author contributed to the writing and synthesis of included reviews. The author(s) read and approved the final manuscript.

\section{Funding}

Corporación Actuemos provided honorariums to the authors (LW, SG, SB) for their contribution to this review. Corporación Actuemos is a Chilean not-forprofit, private organisation that works on sustainable food systems. Corporación Actuemos had no role in this review, nor the decision to submit for publication.

\section{Availability of data and materials}

The datasets used and/or analysed during the umbrella review are available from the corresponding author on reasonable request.

Ethics approval and consent to participate

Not applicable.

\section{Consent for publication}

Not applicable.

\section{Competing interests}

The authors declare that they have no competing interests.

\section{Author details}

${ }^{1}$ School of Medicine and Public Health, The University of Newcastle, Hunter New England Population Health, Locked Bag 10 Wallsend NSW, Newcastle, NSW 2287, Australia. ${ }^{2}$ Corporación Actuemos, Santiago, Chile. ${ }^{3}$ School of Population Health, University of Auckland, Auckland, New Zealand. ${ }^{4}$ Centro de Investigación en Nutrición y Salud, Instituto Nacional de Salud Pública, Cuernavaca, Mexico. ${ }^{5}$ Swinburne University of Technology, School of Health Sciences, Hawthorn, VIC, Australia. 
Received: 23 July 2020 Accepted: 27 October 2020

Published online: 11 January 2021

\section{References}

1. Afshin A, Sur PJ, Fay KA, Cornaby L, Ferrara G, Salama JS, et al. Health effects of dietary risks in 195 countries, 1990-2017: a systematic analysis for the Global Burden of Disease Study 2017. Lancet. 2019;393(10184):1958-72.

2. Lim SS, Vos T, Flaxman AD, Danaei G, Shibuya K, Adair-Rohani H, et al. A comparative risk assessment of burden of disease and injury attributable to 67 risk factors and risk factor clusters in 21 regions, 1990-2010: a systematic analysis for the global burden of disease study 2010. Lancet. 2012;380(9859): 2224-60.

3. World Health Organization. Increasing fruit and vegetable consumption to reduce the risk of noncommunicable diseases. 2019. Available from: https:// www.who.int/elena/titles/fruit_vegetables_ncds/en/.

4. Boeing $H$, Bechthold A, Bub A, Ellinger S, Haller D, Kroke A, et al. Critical review: vegetables and fruit in the prevention of chronic diseases. Eur J Nutr. 2012;51(6):637-63.

5. Li M, Fan Y, Zhang X, Hou W, Tang Z. Fruit and vegetable intake and risk of type 2 diabetes mellitus: meta-analysis of prospective cohort studies. BMJ Open. 2014:4(11):e005497.

6. Slavin JL, Lloyd B. Health benefits of fruits and vegetables. Adv Nutr. 2012; 3(4):506-16

7. Han B, Li X, Yu T. Cruciferous vegetables consumption and the risk of ovarian cancer: a meta-analysis of observational studies. Diagn Pathol. 2014; 9(1):7.

8. Liu B, Mao Q, Cao M, Xie L. Cruciferous vegetables intake and risk of prostate cancer: a meta-analysis. Int J Urol. 2012;19(2):134-41.

9. Liu X, Lv K. Cruciferous vegetables intake is inversely associated with risk of breast cancer: a meta-analysis. Breast. 2013;22(3):309-13.

10. Joint World Health Organisation/FAO Expert Consultation. Diet, nutrition and the prevention of chronic diseases. 2003.

11. Micha R, Khatibzadeh S, Shi P, Andrews KG, Engell RE, Mozaffarian D, et al. Global, regional and national consumption of major food groups in 1990 and 2010: a systematic analysis including 266 country-specific nutrition surveys worldwide. BMJ Open. 2015;5(9):e008705-e.

12. Darfour-Oduro SA, Buchner DM, Andrade JE, Grigsby-Toussaint DS. A comparative study of fruit and vegetable consumption and physical activity among adolescents in 49 Low-and-Middle-Income Countries. Sci Rep. 2018; 8(1):1623.

13. Mason-D'Croz D, Bogard JR, Sulser TB, Cenacchi N, Dunston S, Herrero M, et al. Gaps between fruit and vegetable production, demand, and recommended consumption at global and national levels: an integrated modelling study. Lancet Planet Health. 2019;3(7):e318-e29.

14. Hawkes $\mathrm{C}$, Jewell J, Allen K. A food policy package for healthy diets and the prevention of obesity and diet-related non-communicable diseases: the NOURISHING framework. Obes Rev. 2013;14(S2):159-68.

15. Haddad L, Hawkes C, Waage J, Webb P, Godfray C, Toulmin C. Food systems and diets: Facing the challenges of the 21 st century. London: Global Panel on Agriculture and Food Systems for Nutrition; 2016.

16. World Health Organization. Global nutrition policy review 2016-2017: country progress in creating enabling policy environments for promoting healthy diets and nutrition. 2018.

17. Lachat C, Otchere S, Roberfroid D, Abdulai A, Seret FMA, Milesevic J, et al. Diet and physical activity for the prevention of noncommunicable diseases in low-and middle-income countries: a systematic policy review. Plos Med. 2013;10(6):e1001465.

18. Higgins JPT, Thomas J, Chandler J, Cumpston M, Li T, Page MJ, Welch VA, editors. Cochrane Handbook Syst Rev Intervent. 2nd ed. Chichester: Wiley; 2019.

19. Moher D, Shamseer $L$, Clarke M, Ghersi D, Liberati A, Petticrew M, et al. Preferred reporting items for systematic review and meta-analysis protocols (PRISMA-P) 2015 statement. Syst Rev. 2015:4(1):1.

20. Grimshaw JM, Eccles MP, Lavis JN, Hill SJ, Squires JE. Knowledge translation of research findings. Implement Sci. 2012;7(1):50.

21. Nathan $N$, Janssen $L$, Sutherland R, Hodder RK, Evans CEL, Booth D, et al The effectiveness of lunchbox interventions on improving the foods and beverages packed and consumed by children at Centre-based care or school: a systematic review and meta-analysis. Int J Behav Nutr Phys Activ. 2019;16(1):38.
22. DeCosta $\mathrm{P}$, Moller $\mathrm{P}$, Frost MB, Olsen A. Changing children's eating behaviour - a review of experimental research. Appetite. 2017;113:327-57.

23. Hodder RK, O'Brien KM, Tzelepis F, Wyse RJ, Wolfenden L. Interventions for increasing fruit and vegetable consumption in children aged five years and under. Cochrane Database Syst Rev. 2020;5:CD008552 https://doi.org/10. 1002/14651858.CD008552.pub7.

24. Rueff A, Logomarsino J. Increasing fruit and vegetable intake among manufacturing workers. Int J Workplace Health Manag. 2016;9(1):32-45.

25. Savoie-Roskos MR, Wengreen $H$, Durward $C$. Increasing fruit and vegetable intake among children and youth through gardening-based interventions: a systematic review. J Acad Nutr Diet. 2017;117(2):240-50.

26. Rodriguez Rocha NP, Kim H. eHealth interventions for fruit and vegetable intake: a meta-analysis of effectiveness. Health Educ Behav. 2019:46(6):947-59.

27. Aromataris E, Fernandez R, Godfrey CM, Holly C, Khalil H, Tungpunkom P. Summarizing systematic reviews: methodological development, conduct and reporting of an umbrella review approach. Int J Evid Based Healthc. 2015:13(3):132-40

28. Page MJ, Shamseer L, Altman DG, Tetzlaff J, Sampson M, Tricco AC, et al. Epidemiology and reporting characteristics of systematic reviews of biomedical research: a cross-sectional study. Plos Med. 2016;13(5):e1002028.

29. Authors/Task Force Members, Rydén L, Grant PJ, Anker SD, Berne C, Cosentino $F$, et al. ESC guidelines on diabetes, pre-diabetes, and cardiovascular diseases developed in collaboration with the EASD: the task force on diabetes, prediabetes, and cardiovascular diseases of the European Society of Cardiology (ESC) and developed in collaboration with the European Association for the Study of diabetes (EASD). Eur Heart J. 2013;34(39):3035-87.

30. World Health Organization. Prevention and control of noncommunicable diseases: guidelines for primary health care in low resource settings. Malta: World Health Organization; 2012.

31. Williams B, Mancia G, Spiering W, Agabiti Rosei E, Azizi M, Burnier M, et al. 2018 ESC/ESH guidelines for the management of arterial hypertension: the task force for the management of arterial hypertension of the European Society of Cardiology (ESC) and the European Society of Hypertension (ESH). Eur Heart J. 2018;39(33):3021-104.

32. Cahill K, Stevens S, Perera R, Lancaster T. Pharmacological interventions for smoking cessation: an overview and network meta-analysis. Cochrane Database Syst Rev. 2013;5:CD009329 https://doi.org/10.1002/14651858 CD009329.pub2.

33. Aromataris E MZE. JBI Manual for Evidence Synthesis. JBl; 2020. Available from https://synthesismanual.jbi.global. https://doi.org/10.46658/JBIMES-20-01.

34. Pollock MFR, Becker LA, Pieper D, Hartling L. Chapter V: Overviews of Reviews. In: JPT H, Thomas J, Chandler J, Cumpston M, Li T, Page MJ, Welch VA, editors. Cochrane Handbook for Systematic Reviews of Interventions version 6.0 Cochrane; 2020. Available from www.training.cochrane.org/ handbook. March 2020.

35. Wolfenden L, McCrabb S, Lane C, Barnes C. Interventions to improve fruit and vegetable intake: an overview of systematic reviews; 2020

36. Pollock M, Fernandes RM, Pieper D, Tricco AC, Gates M, Gates A, et al. Preferred reporting items for overviews of reviews (PRIOR): a protocol for development of a reporting guideline for overviews of reviews of healthcare interventions. Syst Rev. 2019;8(1):335.

37. Schultz TJ, Roupas P, Wiechula R, Krause D, Gravier S, Tuckett A, et al. Nutritional interventions for optimizing healthy body composition in older adults in the community: an umbrella review of systematic reviews. JBI Database System Rev Implement Rep. 2016;14(8):257-308.

38. Moher D, Liberati A, Tetzlaff J, Altman DG, Group P. Preferred reporting items for systematic reviews and meta-analyses: the PRISMA statement. Plos Med. 2009;6(7):e1000097.

39. Higgins JPT, Altman DG, Gøtzsche PC, Jüni P, Moher D, Oxman AD, et al. The Cochrane Collaboration's tool for assessing risk of bias in randomised trials. BMJ. 2011;343:d5928.

40. Guyatt GH, Oxman AD, Vist GE, Kunz R, Falck-Ytter $Y$, Alonso-Coello $P$, et al. GRADE: an emerging consensus on rating quality of evidence and strength of recommendations. BMJ. 2008;336(7650):924-6.

41. Guyatt GH, Oxman AD, Vist G, Kunz R, Brozek J, Alonso-Coello P, et al. GRADE guidelines: 4. Rating the quality of evidence-study limitations (risk of bias). J Clin Epidemiol. 2011;64(4):407-15.

42. International WCRF. NOURISHING framework. 2020. Available from: https:// www.wcrf.org/int/policy/policy-databases/nourishing-framework.

43. Afshin A, Penalvo J, Del Gobbo L, Kashaf M, Micha R, Morrish K, et al. CVD prevention through policy: a review of mass media, food/menu labeling, 
taxation/subsidies, built environment, school procurement, worksite wellness, and marketing standards to improve diet. Curr Cardiol Rep. 2015; 17(11):98.

44. Afshin A, Penalvo JL, Del Gobbo L, Silva J, Michaelson M, O'Flaherty M, et al. The prospective impact of food pricing on improving dietary consumption: A systematic review and meta-analysis. Plos One. 2017;12(3):e0172277.

45. Carter P, Bignardi G, Hollands GJ, Marteau TM. Information-based cues at point of choice to change selection and consumption of food, alcohol and tobacco products: a systematic review. BMC Public Health. 2018;18(1):418.

46. Champion KE, Parmenter B, McGowan C, Spring B, Wafford QE, Gardner LA, et al. Effectiveness of school-based eHealth interventions to prevent multiple lifestyle risk behaviours among adolescents: a systematic review and meta-analysis. Lancet Digit Health. 2019;1(5):e206-e21.

47. Cornelsen L, Green R, Turner R, Dangour AD, Shankar B, Mazzocchi M, et al. What happens to patterns of food consumption when food prices change? Evidence from a systematic review and meta-analysis of food Price Elasticities globally. Health Econ. 2015:24(12):1548-59.

48. Feltner C, Peterson K, Palmieri Weber R, Cluff L, Coker-Schwimmer E, Viswanathan $\mathrm{M}$, et al. The effectiveness of Total worker health interventions: a systematic review for a National Institutes of Health pathways to prevention workshop. Ann Intern Med. 2016;165(4):262-9.

49. Girard AW, Self JL, MCAuliffe C, Olude O. The effects of household food production strategies on the health and nutrition outcomes of women and young children: a systematic review. Paediatr Perinat Epidemiol. 2012;26(s1): 205-22.

50. Hendren S, Logomarsino J. Impact of worksite cafeteria interventions on fruit and vegetable consumption in adults: A systematic review. Int J Workplace Health Manage. 2017;10(2):134-52.

51. Hendrie GA, Lease HJ, Bowen J, Baird DL, Cox DN. Strategies to increase children's vegetable intake in home and community settings: a systematic review of literature. Mater Child Nutr. 2017;13(1):e12276.

52. Hollis-Hansen $\mathrm{K}$, Vermont $\mathrm{L}$, Zafron ML, Seidman J, Leone L. The introduction of new food retail opportunities in lower-income communities and the impact on fruit and vegetable intake: a systematic review. Transl Behav Med. 2019;9(5):837-46.

53. Hsiao BS, Sibeko L, Troy LM. A Systematic Review of Mobile Produce Markets: Facilitators and Barriers to Use, and Associations with Reported Fruit and Vegetable Intake. J Acad Nutr Diet. 2019;119(1):76-97.e1.

54. Langford R, Bonell CP, Jones HE, Pouliou T, Murphy SM, Waters E, et al. The WHO health promoting school framework for improving the health and well-being of students and their academic achievement. Cochrane Database Syst Rev. 2014:4:CD008958.

55. Micha R, Karageorgou D, Bakogianni I, Trichia E, Whitsel LP, Story M, et al. Effectiveness of school food environment policies on children's dietary behaviors: A systematic review and meta-analysis. Plos One. 2018;13(3): e0194555.

56. Patnode CD, Evans CV, Senger CA, Redmond N, Lin JS. Behavioral Counseling to Promote a Healthful Diet and Physical Activity for Cardiovascular Disease Prevention in Adults Without Known Cardiovascular Disease Risk Factors: Updated Systematic Review for the U.S. Preventive Services Task Force. Rockville: Agency for Healthcare Research and Quality (US); 2017. Report No.: 15-05222-EF-1. PMID:29364620.

57. Rochira A, Tedesco D, Ubiali A, Fantini MP, Gori D. School gardening activities aimed at obesity prevention improve body mass index and waist circumference parameters in school-aged children: a systematic review and meta-analysis. Child Obes. 2020;16(3):154-73.

58. Silveira JA, Taddei JA, Guerra PH, Nobre MR. Effectiveness of school-based nutrition education interventions to prevent and reduce excessive weight gain in children and adolescents: a systematic review. J Pediatr. 2011;87(5): 382-92.

59. Crockett RA, King SE, Marteau TM, Prevost AT, Bignardi G, Roberts NW, et al. Nutritional labelling for healthier food or non-alcoholic drink purchasing and consumption. Cochrane Database of Syst Rev. 2018;2:CD009315 https:// doi.org/10.1002/14651858.CD009315.pub2.

60. Federici C, Detzel P, Petracca F, Dainelli L, Fattore G. The impact of food reformulation on nutrient intakes and health, a systematic review of modelling studies. BMC Nutr. 2019;5(1):2.

61. World Health Organization. Tackling NCDs: 'best buys' and other recommended interventions for the prevention and control of noncommunicable diseases. Geneva: World Health Organization; 2017. Contract No.: WHO/NMH/NVI/17.9.
62. Assembly UNG. Political declaration of the high-level meeting of the general Assembly on the prevention and control of non-communicable disease. New York: United Nations; 2011.

63. Cobiac $\sqcup$, Tam K, Veerman L, Blakely T. Taxes and Subsidies for Improving Diet and Population Health in Australia: A Cost-Effectiveness Modelling Study. Plos Med. 2017;14(2):-e1002232-e.

64. Rasmussen M, Krølner R, Klepp K-I, Lytle L, Brug J, Bere E, et al. Determinants of fruit and vegetable consumption among children and adolescents: a review of the literature. Part I: quantitative studies. Int J Behav Nutr Phys Act. 2006;3(1):22.

65. Gittelsohn J, Rowan M, Gadhoke P. Interventions in small food stores to change the food environment, improve diet, and reduce risk of chronic disease. Prev Chronic Dis. 2012;9:E59.

\section{Publisher's Note}

Springer Nature remains neutral with regard to jurisdictional claims in published maps and institutional affiliations.

\section{Ready to submit your research? Choose BMC and benefit from:}

- fast, convenient online submission

- thorough peer review by experienced researchers in your field

- rapid publication on acceptance

- support for research data, including large and complex data types

- gold Open Access which fosters wider collaboration and increased citations

- maximum visibility for your research: over $100 \mathrm{M}$ website views per year

At BMC, research is always in progress.

Learn more biomedcentral.com/submissions 